\title{
CONVERGENCY OF THE FUZZY VECTORS IN THE PSEUDO-FUZZY VECTOR SPACE OVER $F_{p}^{1}(1)$
}

\author{
KWEIMEI WU
}

Received 24 March 2003

In 2003, we considered the pseudo-fuzzy vector space SFR over $F_{p}^{1}(1)$. Here, we further discuss the convergency of the fuzzy vectors in SFR.

2000 Mathematics Subject Classification: 08A72.

1. Introduction. In this paper, we discuss the convergency of the fuzzy space over $F_{p}^{1}(1)$ (see [4]). In [4, Section 2], we stated the pseudo-fuzzy vector space SFR over $F_{p}^{1}(1)$ as follows: for two points $P=\left(x^{(1)}, x^{(2)}, \ldots, x^{(n)}\right)$ and $Q=\left(y^{(1)}, y^{(2)}, \ldots, y^{(n)}\right)$ on $\mathbb{R}^{n}$, we have the crisp vector $\overrightarrow{P Q}=\left(y^{(1)}-x^{(1)}, y^{(2)}-x^{(2)}, \ldots, y^{(n)}-x^{(n)}\right)$ in a pseudo-fuzzy vector space $F_{p}^{n}(1)=\left\{\left(a^{(1)}, a^{(2)}, \ldots, a^{(n)}\right)_{1} \forall\left(a^{(1)}, a^{(2)}, \ldots, a^{(n)}\right) \in \mathbb{R}^{n}\right\}$.

There is a one-to-one onto mapping $P=\left(x^{(1)}, x^{(2)}, \ldots, x^{(n)}\right) \leftrightarrow \widetilde{P}=\left(x^{(1)}, x^{(2)}, \ldots\right.$, $\left.x^{(n)}\right)_{1}$. Therefore, for the crisp vector $\overrightarrow{P Q}$, we can define the fuzzy vector $\overrightarrow{\widetilde{P} \widetilde{Q}}=\left(y^{(1)}-\right.$ $\left.x^{(1)}, y^{(2)}-x^{(2)}, \ldots, y^{(n)}-x^{(n)}\right)_{1}=\widetilde{Q} \ominus \widetilde{P}$.

Let the family of the fuzzy sets on $\mathbb{R}^{n}$ satisfying the definitions of convex and normal be $F_{c}$. Obviously, $F_{p}^{n}(1) \subset F_{c}$. Next, we extend the fuzzy vector $\overrightarrow{\widetilde{P} \widetilde{Q}}=\widetilde{Q} \ominus \widetilde{P}$ to $F_{c}$, and $\tilde{X}, \tilde{Y} \in F_{c}$, and define the fuzzy vector $\overrightarrow{\tilde{X}} \vec{Y}=\tilde{Y} \ominus \tilde{X}$. Let SFR $=\left\{\overrightarrow{\tilde{X}} \tilde{Y} \forall \tilde{X}, \tilde{Y} \in F_{c}\right\}$. Then we have the pseudo-fuzzy vector space over $F_{p}^{n}(1)\left(=a_{1} \forall a \in \mathbb{R}\right)$. In Section 3, we will discuss the convergency of the fuzzy vectors in SFR.

2. Preparation. In [4], we discussed the pseudo-fuzzy vector space SFR over $F_{p}^{1}(1)$. In order to discuss the convergence of the fuzzy vectors in SFR, we need to know some definitions.

Definition 2.1. ( $\left.1^{\circ}\right)$ The fuzzy set $\tilde{A}$ on $\mathbb{R}=(-\infty, \infty)$ is convex if and only if every ordinary set $A(\alpha)=\left\{x \mid \mu_{\widetilde{A}}(x) \geq \alpha \forall \alpha \in[0,1]\right\}$ is convex, and hence $A(\alpha)$ is a closed interval of $\mathbb{R}$.

$\left(2^{\circ}\right)$ The fuzzy set $\tilde{A}$ on $\mathbb{R}$ is normal if and only if $\bigvee_{x \in \mathbb{R}} \mu_{\tilde{A}}(x)=1$.

Next, we extend this definition to $\mathbb{R}^{n}$ by saying that the membership function of the fuzzy set $\widetilde{D}$ on $\mathbb{R}^{n}$ is $\mu_{\widetilde{D}}\left(x^{(1)}, x^{(2)}, \ldots, x^{(n)}\right) \in[0,1]$ for all $\left(x^{(1)}, x^{(2)}, \ldots, x^{(n)}\right) \in \mathbb{R}^{n}$.

DeFinition 2.2. The $\alpha$-cut $(0 \leq \alpha \leq 1)$ of the fuzzy set $\tilde{D}$ on $\mathbb{R}^{n}$ is defined by $D(\alpha)=\left\{\left(x^{(1)}, x^{(2)}, \ldots, x^{(n)}\right) \mid \mu_{\tilde{D}}\left(x^{(1)}, x^{(2)}, \ldots, x^{(n)}\right) \geq \alpha\right\}$.

DEFINITION 2.3. ( $\left.1^{\circ}\right)$ The fuzzy set $\widetilde{D}$ on $\mathbb{R}^{n}$ is convex if and only if every ordinary set $D(\alpha)=\left\{\left(x^{(1)}, x^{(2)}, \ldots, x^{(n)}\right) \mid \mu_{\tilde{D}}\left(x^{(1)}, x^{(2)}, \ldots, x^{(n)}\right) \geq \alpha \forall \alpha \in[0,1]\right\}$ is a convex closed subset of $\mathbb{R}^{n}$. 
$\left(2^{\circ}\right)$ The fuzzy set $\tilde{D}$ is normal if and only if $\bigvee_{\left(x^{(1)}, x^{(2)}, \ldots, x^{(n)}\right) \in \mathbb{R}^{n}} \mu_{\widetilde{D}}\left(x^{(1)}, x^{(2)}, \ldots\right.$, $\left.x^{(n)}\right)=1$.

Let the family of the fuzzy sets on $\mathbb{R}^{n}$ satisfying Definition $2.3\left(1^{\circ}\right),\left(2^{\circ}\right)$ be $F_{c}$.

Definition 2.4 (Pu and Liu [3]). The fuzzy set $a_{\alpha}(0 \leq \alpha \leq 1)$ on $\mathbb{R}$ is called a level $\alpha$ fuzzy point on $\mathbb{R}$ if its membership function $\mu_{a_{\alpha}}(x)$ is

$$
\mu_{a_{\alpha}}(x)= \begin{cases}\alpha, & x=a \\ 0, & x \neq a\end{cases}
$$

Let the family of all level $\alpha$ fuzzy points on $\mathbb{R}$ be $F_{p}^{(1)}(\alpha)=\left\{a_{\alpha} \forall \alpha \in \mathbb{R}\right\}, 0 \leq \alpha \leq 1$.

Definition 2.5. The fuzzy set $\left(a^{(1)}, a^{(2)}, \ldots, a^{(n)}\right)_{\alpha}(0 \leq \alpha \leq 1)$ is called a level $\alpha$ fuzzy point on $\mathbb{R}^{n}$ if its membership function is

$$
\begin{aligned}
& \mu_{\left(a^{(1)}, a^{(2)}, \ldots, a^{(n)}\right)_{\alpha}}\left(x^{(1)}, x^{(2)}, \ldots, x^{(n)}\right) \\
& \quad= \begin{cases}\alpha, & \text { if }\left(x^{(1)}, x^{(2)}, \ldots, x^{(n)}\right)=\left(a^{(1)}, a^{(2)}, \ldots, a^{(n)}\right), \\
0, & \text { elsewhere. }\end{cases}
\end{aligned}
$$

Let the family of all level $\alpha$ fuzzy points on $\mathbb{R}^{n}$ be

$$
\begin{gathered}
F_{p}^{(n)}(\alpha)=\left\{\left(a^{(1)}, a^{(2)}, \ldots, a^{(n)}\right)_{\alpha} \forall\left(a^{(1)}, a^{(2)}, \ldots, a^{(n)}\right) \in \mathbb{R}^{n}\right\}, \quad 0 \leq \alpha \leq 1, \\
F_{p}^{(n)}=\bigcup_{0 \leq \alpha \leq 1} F_{p}^{(n)}(\alpha) .
\end{gathered}
$$

For each $a_{\alpha} \in F_{p}^{1}(\alpha)$, regard $a_{\alpha}=(a, a, \ldots, a)_{\alpha}$ as a special level $\alpha$ fuzzy point on $\mathbb{R}^{n}$ degenerated from a level $\alpha$ fuzzy point $\left(a^{(1)}, a^{(2)}, \ldots, a^{(n)}\right)$ with $a^{(1)}=a^{(2)}=\cdots=$ $a^{(n)}=a$. Hence, we have the following expression:

$$
\begin{aligned}
\mu_{(a, a, \ldots, a)_{\alpha}}\left(x^{(1)}, x^{(2)}, \ldots, x^{(n)}\right) & = \begin{cases}\alpha, & \left(x^{(1)}, x^{(2)}, \ldots, x^{(n)}\right)=(a, a, \ldots, a), \\
0, & \left(x^{(1)}, x^{(2)}, \ldots, x^{(n)}\right) \neq(a, a, \ldots, a),\end{cases} \\
& =\mu_{a_{\alpha}}\left(x^{(1)}, x^{(2)}, \ldots, x^{(n)}\right) .
\end{aligned}
$$

Definition 2.6. For $D \subset \mathbb{R}^{n}$, call $D_{\alpha}, 0 \leq \alpha \leq 1$, a level $\alpha$ fuzzy domain on $\mathbb{R}^{n}$ if its membership function is

$$
\mu_{D_{\alpha}}\left(x^{(1)}, x^{(2)}, \ldots, x^{(n)}\right)= \begin{cases}\alpha, & \text { if }\left(x^{(1)}, x^{(2)}, \ldots, x^{(n)}\right) \in D, \\ 0, & \text { if }\left(x^{(1)}, x^{(2)}, \ldots, x^{(n)}\right) \notin D .\end{cases}
$$

Let the family of all the level $\alpha$ fuzzy domains on $\mathbb{R}^{n}$ be $F D^{*}=\left\{E_{\alpha} \forall E \subset \mathbb{R}^{n}\right\}$, and let the family of all subsets of $\mathbb{R}^{n}$ be $\mathscr{P}\left(\mathbb{R}^{n}\right)=\left\{E \forall E \subset \mathbb{R}^{n}\right\}$. 
Then there is a one-to-one mapping $\eta$ between $\mathscr{P}\left(\mathbb{R}^{n}\right)$ and $F D^{*}$ :

$$
\begin{gathered}
E \in \mathscr{P}\left(\mathbb{R}^{n}\right) \longleftrightarrow \eta(E)=E_{\alpha} \in F D^{*}, \\
\eta^{(-1)}\left(E_{\alpha}\right)=E, \quad \alpha \in[0,1] .
\end{gathered}
$$

Since $\tilde{D} \in F_{\mathcal{C}}$, the $\alpha$-cut $D(\alpha)(0 \leq \alpha \leq 1)$ of $\tilde{D}$ can be mapped to $D(\alpha)_{\alpha}$.

Thus, we have the following decomposition principle:

$$
\forall \tilde{D} \in F_{c}, \quad \tilde{D}=\bigcup_{\alpha \in[0,1]} D(\alpha)_{\alpha}
$$

From Kaufmann and Gupta [2], we have for $D, E \subset \mathbb{R}^{n}, k \in \mathbb{R}$,

$$
\begin{aligned}
& D(+) E=\left\{\left(x^{(1)}+y^{(1)}, x^{(2)}+y^{(2)}, \ldots, x^{(n)}+y^{(n)}\right)\right. \\
& \left.\forall\left(x^{(1)}, x^{(2)}, \ldots, x^{(n)}\right) \in D,\left(y^{(1)}, y^{(2)}, \ldots, y^{(n)}\right) \in E\right\}, \\
& D(-) E=\left\{\left(x^{(1)}-y^{(1)}, x^{(2)}-y^{(2)}, \ldots, x^{(n)}-y^{(n)}\right)\right. \\
& \left.\forall\left(x^{(1)}, x^{(2)}, \ldots, x^{(n)}\right) \in D,\left(y^{(1)}, y^{(2)}, \ldots, y^{(n)}\right) \in E\right\}, \\
& k(\cdot) D=\left\{\left(k x^{(1)}, k x^{(2)}, \ldots, k x^{(n)}\right) \forall\left(x^{(1)}, x^{(2)}, \ldots, x^{(n)}\right) \in D\right\} \text {. }
\end{aligned}
$$

From (2.6), (2.7), (2.8), (2.9), (2.10), and the definition of the $\alpha$-cut, we have that

(i) the $\alpha$-cut of $\widetilde{D}(+) \widetilde{E}$ is $D(\alpha)+E(\alpha)$,

$$
\widetilde{D} \oplus \widetilde{E}=\bigcup_{0 \leq \alpha \leq 1}(D(\alpha)(+) E(\alpha))_{\alpha}
$$

(ii) the $\alpha$-cut of $\widetilde{D}(-) \widetilde{E}$ is $D(\alpha)-E(\alpha)$,

$$
\tilde{D} \ominus \widetilde{E}=\bigcup_{0 \leq \alpha \leq 1}(D(\alpha)(-) E(\alpha))_{\alpha}
$$

(iii) the $\alpha$-cut of $k_{1} \odot w t D$ is $k(\cdot) D(\alpha)$,

$$
k_{1} \odot \widetilde{D}=\bigcup_{0 \leq \alpha \leq 1}(k(\cdot) D(\alpha))_{\alpha}
$$

In the crisp case on $\mathbb{R}^{n}$, we can consider the $n$-dimensional vector space $E^{n}$ over $\mathbb{R}$.

Let $P=\left(p^{(1)}, p^{(2)}, \ldots, p^{(n)}\right), Q=\left(q^{(1)}, q^{(2)}, \ldots, q^{(n)}\right), A=\left(a^{(1)}, a^{(2)}, \ldots, a^{(n)}\right), B=\left(b^{(1)}\right.$, $\left.b^{(2)}, \ldots, b^{(n)}\right) \in \mathbb{R}^{n} ; k \in \mathbb{R}$.

Define the crisp vectors $\overrightarrow{P Q}, \overrightarrow{A B}+\overrightarrow{P Q}$, and $k \cdot \overrightarrow{P Q}$ as follows:

$$
\begin{aligned}
& \overrightarrow{P Q}=\left(q^{(1)}-p^{(1)}, q^{(2)}-p^{(2)}, \ldots, q^{(n)}-p^{(n)}\right)=Q(-) P, \\
& \overrightarrow{A B}+\overrightarrow{P Q}=\left(b^{(1)}+q^{(1)}-a^{(1)}-p^{(1)}, b^{(2)}+q^{(2)}-a^{(2)}-p^{(2)},\right. \\
&\left.\ldots, b^{(n)}+q^{(n)}-a^{(n)}-p^{(n)}\right), \\
& k \cdot \overrightarrow{P Q}=\left(k q^{(1)}-k p^{(1)}, k q^{(2)}-k p^{(2)}, \ldots, k q^{(n)}-k p^{(n)}\right) .
\end{aligned}
$$


Let $O=(0,0, \ldots, 0) \in \mathbb{R}^{n}, \overrightarrow{O P}=\left(p^{(1)}, p^{(2)}, \ldots, p^{(n)}\right), \overrightarrow{O O}=(0,0, \ldots, 0)$, and let $E^{n}=$ $\left\{\overrightarrow{P Q}=\left(q^{(1)}-p^{(1)}, q^{(2)}-p^{(2)}, \ldots, q^{(n)}-p^{(n)}\right) \forall P, Q \in \mathbb{R}^{n}\right\}$. This is an $n$-dimensional vector space over $\mathbb{R}$. There is a one-to-one onto mapping between the point $\left(a^{(1)}, a^{(2)}\right.$, $\ldots, a^{n}$ ) on $\mathbb{R}^{n}$ and the level 1 fuzzy point $\left(a^{(1)}, a^{(2)}, \ldots, a^{n}\right)_{1}$ on $F_{p}^{n}(1)$ :

$$
\begin{aligned}
& \rho:\left(a^{(1)}, a^{(2)}, \ldots, a^{(n)}\right) \in \mathbb{R}^{n} \longleftrightarrow \rho\left(a^{(1)}, a^{(2)}, \ldots, a^{(n)}\right) \\
& \quad=\left(a^{(1)}, a^{(2)}, \ldots, a^{(n)}\right)_{1} \in F_{p}^{n}(1) .
\end{aligned}
$$

Let $\widetilde{P}=\left(p^{(1)}, p^{(2)}, \ldots, p^{(n)}\right)_{1}, \widetilde{Q}=\left(q^{(1)}, q^{(2)}, \ldots, q^{(n)}\right)_{1} \in F_{p}^{n}(1)$. From (2.14) and (2.17), we have the following definition:

$$
\overrightarrow{\widetilde{P}} \widetilde{Q}=\left(q^{(1)}-p^{(1)}, q^{(2)}-p^{(2)}, \ldots, q^{(n)}-p^{(n)}\right)_{1}=\widetilde{Q} \ominus \widetilde{P} .
$$

Let $F E^{n}=\left\{\overrightarrow{\widetilde{P} \widetilde{Q}} \forall \widetilde{P}, \widetilde{Q} \in F_{p}^{n}(1)\right\}$. From (2.14) and (2.18), we have the one-to-one onto mappings

$$
\begin{aligned}
\overrightarrow{P Q}= & \left(q^{(1)}-p^{(1)}, q^{(2)}-p^{(2)}, \ldots, q^{(n)}-p^{(n)}\right) \\
\longleftrightarrow & \rho(\overrightarrow{P Q})=\left(q^{(1)}-p^{(1)}, q^{(2)}-p^{(2)}, \ldots, q^{(n)}-p^{(n)}\right)_{1} \\
= & \overrightarrow{\widetilde{P}} \in F E^{n}, \\
\overrightarrow{A B}+\overrightarrow{P Q}= & \left(b^{(1)}+q^{(1)}-a^{(1)}-p^{(1)}, b^{(2)}+q^{(2)}-a^{(2)}-p^{(2)},\right. \\
& \left.\ldots, b^{(n)}+q^{(n)}-a^{(n)}-p^{(n)}\right) \\
\longleftrightarrow & \left(b^{(1)}+q^{(1)}-a^{(1)}-p^{(1)}, b^{(2)}+q^{(2)}-a^{(2)}-p^{(2)},\right. \\
& \left.\quad \ldots, b^{(n)}+q^{(n)}-a^{(n)}-p^{(n)}\right)_{1} \\
= & \overrightarrow{\widetilde{A} \widetilde{B}} \oplus \overrightarrow{\widetilde{P} \widetilde{Q}} \\
k \cdot \overrightarrow{P Q}= & \left(k q^{(1)}-k p^{(1)}, k q^{(2)}-k p^{(2)}, \ldots, k q^{(n)}-k p^{(n)}\right) \\
\longleftrightarrow & \left(k q^{(1)}-k p^{(1)}, k q^{(2)}-k p^{(2)}, \ldots, k q^{(n)}-k p^{(n)}\right)_{1} \\
= & k{ }_{1} \odot \overrightarrow{\widetilde{P} \widetilde{Q}} .
\end{aligned}
$$

Therefore, $F E^{n}=\left\{\overrightarrow{\widetilde{P} \widetilde{Q}} \forall \widetilde{P}, \widetilde{Q} \in F_{p}^{n}(1)\right\}$ is a vector space over $F_{p}^{n}(1)$ in fuzzy sense.

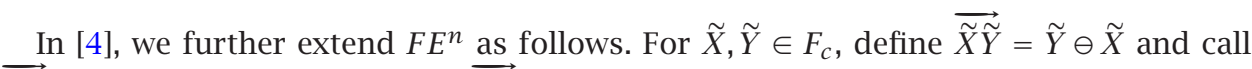
$\overrightarrow{\tilde{X} \tilde{Y}}$ a fuzzy vector. Let SFR $=\left\{\overrightarrow{\tilde{X}} \tilde{Y} \forall \tilde{X}, \tilde{Y} \in F_{c}\right\}$. In [4], we proved that the following properties hold. 
Property 2.7. For $\overrightarrow{\tilde{X} \tilde{Y}}, \overrightarrow{\widetilde{W}} \widetilde{Z} \in \mathrm{SFR}$,

$$
\overrightarrow{\tilde{X} \tilde{Y}}=\overrightarrow{\widetilde{W} \widetilde{Z}} \Longleftrightarrow \tilde{Y} \ominus \tilde{X}=\tilde{Z} \ominus \widetilde{W} .
$$

Property 2.8. For $\overrightarrow{\tilde{X}} \widetilde{Y}, \overrightarrow{\widetilde{W}} \widetilde{Z} \in \mathrm{SFR}, k \in \mathbb{R}$,

(1 $\left.{ }^{\circ}\right) \overrightarrow{\tilde{X} \tilde{Y}} \oplus \overrightarrow{\widetilde{W} \widetilde{Z}}=\overrightarrow{\widetilde{A} \widetilde{B}}$, where $\tilde{A}=\tilde{X} \oplus \widetilde{W}, \widetilde{B}=\tilde{Y} \oplus \tilde{Z}$;

$\left(2^{\circ}\right) k_{1} \odot \overrightarrow{\tilde{X} \tilde{Y}}=\overrightarrow{\widetilde{C}} \widetilde{D}$, where $\widetilde{C}=k_{1} \odot \tilde{X}, \widetilde{D}=k_{1} \odot \tilde{Y}$.

Property 2.9. For $\overrightarrow{\tilde{X}} \widetilde{\tilde{Y}}, \overrightarrow{\widetilde{W}} \widetilde{Z}, \overrightarrow{\widetilde{U} \tilde{V}} \in \mathrm{SFR}, k, t \in \mathbb{R}$,

$\left(1^{\circ}\right) \overrightarrow{\tilde{X} \tilde{Y}} \oplus \overrightarrow{\widetilde{W} \widetilde{Z}}=\overrightarrow{\widetilde{W} \widetilde{Z}} \oplus \overrightarrow{\tilde{X} \tilde{Y}}$

$\left(2^{\circ}\right)(\overrightarrow{\tilde{X}} \vec{Y} \oplus \overrightarrow{\widetilde{W} \tilde{Z}}) \oplus \overrightarrow{\tilde{U} \tilde{V}}=\overrightarrow{\tilde{X} \tilde{Y}} \oplus(\overrightarrow{\widetilde{W} \tilde{Z}} \oplus \overrightarrow{\tilde{U}} \vec{V})$;

$\left(^{\circ}\right) \overrightarrow{\tilde{X} \tilde{Y}} \oplus \overrightarrow{\widetilde{O} \widetilde{O}}=\overrightarrow{\tilde{X}} \vec{Y}$, where $\overrightarrow{\widetilde{O} \widetilde{O}}=(0,0, \ldots, 0)_{1}$;

$\left(4^{\circ}\right) k_{1} \odot\left(\underline{t_{1} \odot} \overrightarrow{\tilde{\tilde{X}} \tilde{Y}}\right)=(k t)_{1} \odot \overrightarrow{\tilde{X} \tilde{Y}}$;

$\left(5^{\circ}\right) k_{1} \odot(\overrightarrow{\tilde{X} \tilde{Y}} \oplus \overrightarrow{\widetilde{W} \widetilde{Z}})=\left(k_{1} \odot \overrightarrow{\tilde{X} \tilde{Y}}\right) \oplus\left(k_{1} \odot \overrightarrow{\widetilde{W} \widetilde{Z}}\right)$;

$\left(6^{\circ}\right) 1 \odot \overrightarrow{\tilde{X} \tilde{Y}}=\overrightarrow{\tilde{X}} \tilde{Y}$.

In SFR, the following do not hold.

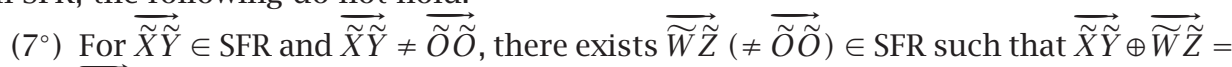
$\overrightarrow{\widetilde{O}}$

$\left(8^{\circ}\right)(k+t)_{1} \odot \overrightarrow{\tilde{X} \tilde{Y}}=\left(k_{1} \odot \overrightarrow{\tilde{X}} \tilde{Y}\right) \oplus\left(t_{1} \odot \overrightarrow{\tilde{X} \tilde{Y}}\right)$.

From Property 2.9, we know that SFR satisfies all the conditions that the vector space required, except $\left(7^{\circ}\right)$ and $\left(8^{\circ}\right)$. Therefore, in [4], we called SFR a pseudo-fuzzy vector space over $F_{p}^{1}(1)$.

EXAMPLE 2.10 (a moving station carrying a missile on it). This car left from point $P=(2,5)$ passing through point $Q=(4,6)$, arrived at $R=(8,9)$, and aiming at the target $Z=(100,200)$. As we can see, the missile usually falls in the vicinity of $Z$, say $\tilde{Z}$, instead of hitting at $Z$ exactly.

Let the membership function of $\tilde{Z}$ be

$$
\mu_{\tilde{Z}}\left(x^{(1)}, x^{(2)}\right)= \begin{cases}\frac{1}{25}\left(25-\left(x^{(1)}-100\right)^{2}-\left(x^{(2)}-200\right)^{2}\right), \\ & \text { if } \left.\left(x^{(1)}-100\right)^{2}+\left(x^{(2)}-200\right)^{2}\right) \leq 25, \\ 0, & \text { elsewhere. }\end{cases}
$$

Consider the level 1 fuzzy points $\widetilde{P}=(2,5)_{1}, \widetilde{Q}=(4,6)_{1}$, and $\widetilde{R}=(8,9)_{1}$. We have the fuzzy routes

$$
\widetilde{P} \longrightarrow \widetilde{Q} \longrightarrow \widetilde{R} \longrightarrow \tilde{Z}
$$


and hence the fuzzy vectors $\overrightarrow{\widetilde{P} \widetilde{Q}}=(2,1)_{1}, \overrightarrow{\widetilde{Q} \widetilde{R}}=(4,3)_{1}, \overrightarrow{\widetilde{R} \widetilde{Z}}=\tilde{Z} \ominus \widetilde{R}$, and $\overrightarrow{\widetilde{P} \widetilde{Z}}=\tilde{Z} \ominus \widetilde{P}$. By extension theory, the membership function of $\overrightarrow{\widetilde{R} \widetilde{Z}}=\tilde{Z} \ominus \widetilde{R}$ is

$$
\begin{aligned}
\mu_{\widetilde{R} \tilde{Z}}\left(z^{(1)}, z^{(2)}\right) & =\sup _{z^{(j)}=v^{(j)}-u^{(j)}, j=1,2} \mu_{\widetilde{R}}\left(u^{(1)}, u^{(2)}\right) \wedge \mu_{\widetilde{Z}}\left(v^{(1)}, v^{(2)}\right) \\
& =\mu_{\widetilde{Z}}\left(z^{(1)}+8, z^{(2)}+9\right) \\
& = \begin{cases}\frac{1}{25}\left(25-\left(z^{(1)}-92\right)^{2}-\left(z^{(2)}-191\right)^{2}\right), \\
\text { if }\left(z^{(1)}-92\right)^{2}+\left(z^{(2)}-191\right)^{2} \leq 25, \\
0, & \text { elsewhere. }\end{cases}
\end{aligned}
$$

Similarly,

$$
\mu_{\overrightarrow{\widetilde{P} \tilde{Z}}}\left(z^{(1)}, z^{(2)}\right)= \begin{cases}\frac{1}{25}(25- & \left.\left(z^{(1)}-98\right)^{2}-\left(z^{(2)}-195\right)^{2}\right), \\ & \text { if }\left(z^{(1)}-98\right)^{2}+\left(z^{(2)}-195\right)^{2} \leq 25, \\ & \text { elsewhere. }\end{cases}
$$

Let $S=(98,202)$. It is clear that $(98,202)$ is within the circle of center $(100,200)$ and radius 5. The crisp vector which starts with the point $P=(2,5)$ and ends at $S=(98,202)$ is $\overrightarrow{P S}=(96,197)$. Its grade of membership in $\overrightarrow{\widetilde{P} \widetilde{Z}}$ from $(2.23)$ is $\mu_{\overrightarrow{\widetilde{P}} \tilde{Z}}(96,197)=(1 / 25)(25-$ $\left.2^{2}-2^{2}\right)=0.68$, that is, the grade of membership of the fuzzy vector $\overrightarrow{\widetilde{P} \widetilde{Z}}$ for the crisp vector $\overrightarrow{P S}$ is 0.68 . Let the aim be $T=(100,200)$. The crisp vector beginning at $\underline{P}=(2,5)$ and aiming at $T=(100,200)$ is $\overrightarrow{P T}=(98,195)$. Its grade of membership in $\vec{P} \widetilde{Z}$, again from $(2.23)$, is $\mu_{\vec{P} \tilde{Z}}(98,195)=(1 / 25)\left(25-0^{2}-0^{2}\right)=1$, that is, the grade of membership of the fuzzy vector $\overrightarrow{\widetilde{P}} \widetilde{Z}$ for the crisp vector $\overrightarrow{P T}$ is 1 .

EXAMPLE 2.11. In a shooting practice, let $C((10,30), 1+1 / m)=\left\{(x, y) \mid(x-10)^{2}+\right.$ $\left.(y-30)^{2} \leq(1+1 / m)^{2}\right\}$, always shooting at $(1,2)$ and aiming at $Z=(10,30)$. At the first time, the bullet was falling in $C((10,30), 2(=1+1))$. At the second time, it was falling in $C((10,30), 1+1 / 2)$. At the $m$ th time, it was falling in $C((10,30), 1+1 / m)$. In other words, the bullet was more and more closer to $C((10,30), 1)$, that is, more and more accurate.

Let the fuzzy aim be $\widetilde{Z}_{m}$, its membership function is

$$
\mu_{\widetilde{Z}_{m}}=\left\{\begin{array}{l}
\frac{1}{(1+1 / m)^{2}}\left[\left(1+\frac{1}{m}\right)^{2}-(x-10)^{2}-(y-30)^{2}\right], \\
\quad \text { if }(x-10)^{2}+(y-30)^{2} \leq\left(1+\frac{1}{m}\right)^{2}, \\
\quad \text { elsewhere. }
\end{array}\right.
$$

Thus, we have the $m$ th fuzzy vector $\overrightarrow{\widetilde{Q} \widetilde{Z}_{m}}, m=1,2, \ldots$, where $\widetilde{Q}=(1,2)_{1}$. In the next section, we will discuss the convergency of the fuzzy vectors in SFR and find out the limit fuzzy vector $\lim _{n \rightarrow \infty} \overrightarrow{\widetilde{Q} \widetilde{Z}_{m}}$. 
3. The convergency of the vectors in SFR. Before we try to investigate the convergency of the fuzzy vectors in SFR, we first define the following open set in $\mathbb{R}^{n}$ and discuss some properties (Properties 3.4, 3.7, 3.10, 3.11, 3.12, 3.13, 3.14, 3.15, 3.16, and 3.17). Let

$$
\begin{aligned}
& O\left(\left(a^{(1,1)}, a^{(1,2)}\right), \ldots,\left(a^{(n, 1)}, a^{(n, 2)}\right)\right) \\
& \quad=\left\{\left(x^{(1)}, x^{(2)}, \ldots, x^{(n)}\right) \mid a^{(j, 1)}<x^{(j)}<a^{(j, 2)}, j=1,2, \ldots, n\right\} .
\end{aligned}
$$

From (2.8), (2.9), and (2.10), we have

$$
\begin{aligned}
& O\left(\left(a^{(1,1)}, a^{(1,2)}\right), \ldots,\left(a^{(n, 1)}, a^{(n, 2)}\right)\right)(+) O\left(\left(b^{(1,1)}, b^{(1,2)}\right), \ldots,\left(b^{(n, 1)}, b^{(n, 2)}\right)\right) \\
&=\left\{\left(z^{(1)}, z^{(2)}, \ldots, z^{(n)}\right) \mid z^{(j)}=x^{(j)}+y^{(j)}, a^{(j, 1)}<x^{(j)}<a^{(j, 2)},\right. \\
&\left.b^{(j, 1)}<y^{(j)}<b^{(j, 2)}, j=1,2, \ldots, n\right\} \\
&= O\left(\left(a^{(1,1)}+b^{(1,1)}, a^{(1,2)}+b^{(1,2)}\right), \ldots,\left(a^{(n, 1)}+b^{(n, 1)}, a^{(n, 2)}+b^{(n, 2)}\right)\right), \\
& O\left(\left(a^{(1,1)}, a^{(1,2)}\right), \ldots,\left(a^{(n, 1)}, a^{(n, 2)}\right)\right)(-) O\left(\left(b^{(1,1)}, b^{(1,2)}\right), \ldots,\left(b^{(n, 1)}, b^{(n, 2)}\right)\right) \\
&=\left\{\left(z^{(1)}, z^{(2)}, \ldots, z^{(n)}\right) \mid z^{(j)}=x^{(j)}-y^{(j)}, a^{(j, 1)}<x^{(j)}<a^{(j, 2)},\right. \\
&\left.\quad b^{(j, 1)}<y^{(j)}<b^{(j, 2)}, j=1,2, \ldots, n\right\} \\
&=O\left(\left(a^{(1,1)}-b^{(1,1)}, a^{(1,2)}-b^{(1,2)}\right), \ldots,\left(a^{(n, 1)}-b^{(n, 1)}, a^{(n, 2)}-b^{(n, 2)}\right)\right) .
\end{aligned}
$$

If $k>0$,

$$
\begin{aligned}
k(\cdot) & O\left(\left(a^{(1,1)}, a^{(1,2)}\right), \ldots,\left(a^{(n, 1)}, a^{(n, 2)}\right)\right) \\
& =\left\{\left(z^{(1)}, z^{(2)}, \ldots, z^{(n)}\right) \mid z^{(j)}=k x^{(j)}, a^{(j, 1)}<x^{(j)}<a^{(j, 2)}, j=1,2, \ldots, n\right\} \\
& =O\left(\left(k a^{(1,1)}, k a^{(1,2)}\right), \ldots,\left(k a^{(n, 1)}, k a^{(n, 2)}\right)\right) .
\end{aligned}
$$

If $k<0$,

$$
\begin{aligned}
k(\cdot) & O\left(\left(a^{(1,1)}, a^{(1,2)}\right), \ldots,\left(a^{(n, 1)}, a^{(n, 2)}\right)\right) \\
& =\left\{\left(z^{(1)}, z^{(2)}, \ldots, z^{(n)}\right) \mid z^{(j)}=k x^{(j)}, a^{(j, 1)}<x^{(j)}<a^{(j, 2)}, j=1,2, \ldots, n\right\} \\
& =O\left(\left(k a^{(1,2)}, k a^{(1,1)}\right), \ldots,\left(k a^{(n, 2)}, k a^{(n, 1)}\right)\right) .
\end{aligned}
$$

Let $\mathscr{B}=\left\{O\left(\left(a^{(1,1)}, a^{(1,2)}\right), \ldots,\left(a^{(n, 1)}, a^{(n, 2)}\right)\right)_{\alpha} \forall a^{(j, 1)}<a^{(j, 2)}, a^{(j, 1)}, a^{(j, 2)} \in \mathbb{R}, j=\right.$ $1,2, \ldots, n ; 0 \leq \alpha \leq 1\}$.

Let $\mathscr{B}^{*}$ be the family of fuzzy sets in $\mathscr{B}$ or any arbitrary unions of these fuzzy sets.

REMARK 3.1. Any intersection of two fuzzy sets in $\mathscr{B}$ belongs to $\mathscr{B}$, and when two fuzzy sets in $\mathscr{B}$ have no intersection, we call their intersection $\varnothing$.

From (2.3), let $F=F_{p}^{n} \cup F_{c} \cup \mathscr{B}^{*}$. In order to consider the problem of convergency, we first consider the topology for $F$. 
DEFINITION 3.2. $\widetilde{Q} \in F$ is an open fuzzy set if and only if for each $\left(x^{(1)}, x^{(2)}, \ldots, x^{(n)}\right)_{\alpha}$ $\subset \widetilde{Q}$, there exists $\widetilde{O} \in \mathscr{B}$ such that $\left(x^{(1)}, x^{(2)}, \ldots, x^{(n)}\right)_{\alpha} \subset \widetilde{O} \subset \widetilde{Q}$.

Let $T_{F}$ be the family of all open fuzzy sets satisfying Definition 3.2. Obviously, $\mathscr{B}^{*} \subset$ $T_{F}$.

DEFINITION 3.3 (Chang [1]). $T$ is a family of fuzzy sets in the space $X$ satisfying the following:

$\left(1^{\circ}\right) \varnothing, X \in T$,

$\left(2^{\circ}\right) \tilde{A}, \widetilde{B} \in T$, then $\tilde{A} \cap \widetilde{B} \in T$,

( $\left.3^{\circ}\right) \tilde{A}_{j} \in T, j \in I$ (any index set), then $\bigcup_{j \in I} \tilde{A}_{j} \in T$.

$T$ is called a fuzzy topology for $X$ and $(X, T)$ is called a fuzzy topological space (abbreviated as FTS).

Property 3.4. $T_{F}$ is a fuzzy topology for $\mathbb{R}^{n},\left(\mathbb{R}^{n}, T_{F}\right)$ are fuzzy topological sets in $\mathbb{R}^{n}$ that are restricted in $F$.

Proof. $\left(1^{\circ}\right)$ It is obvious that $\mathbb{R}^{n} \in T_{F}$. Definition $3.3\left(1^{\circ}\right)$ is fulfilled.

$\left(2^{\circ}\right)$ For $\widetilde{D}, \widetilde{E} \in T_{F}$ and $\left(x^{(1)}, x^{(2)}, \ldots, x^{(n)}\right)_{\alpha} \subset \widetilde{D} \cap \widetilde{E}$, we have $\left(x^{(1)}, x^{(2)}, \ldots, x^{(n)}\right)_{\alpha} \subset$ $\widetilde{D}$ and $\left(x^{(1)}, x^{(2)}, \ldots, x^{(n)}\right)_{\alpha} \subset \widetilde{E}$. From Definition 3.2, there exist $\widetilde{I}, \tilde{J} \in \mathscr{B}$ such that $\left(x^{(1)}, x^{(2)}, \ldots, x^{(n)}\right)_{\alpha} \subset \tilde{I} \subset \widetilde{D}$ and $\left(x^{(1)}, x^{(2)}, \ldots, x^{(n)}\right)_{\alpha} \subset \tilde{J} \subset \widetilde{E}$. Therefore, $\left(x^{(1)}, x^{(2)}, \ldots\right.$, $\left.x^{(n)}\right)_{\alpha} \subset \tilde{I} \cap \tilde{J}$. Hence, $\tilde{I} \cap \tilde{J} \subset \tilde{D} \cap \widetilde{E}$. Thus, $\tilde{D} \cap \widetilde{E} \in T_{F}$. Definition 3.3(2 $\left.2^{\circ}\right)$ is fulfilled.

$\left(3^{\circ}\right)$ For $\widetilde{D}_{j} \in T_{F}, j \in I$, and each $\left(x^{(1)}, x^{(2)}, \ldots, x^{(n)}\right)_{\alpha} \subset \bigcup_{j \in I} \widetilde{D}_{j}$, there exists $m \in$ $I$ such that $\left(x^{(1)}, x^{(2)}, \ldots, x^{(n)}\right)_{\alpha} \subset \widetilde{D}_{m}$. By Definition 3.2, there is a $\widetilde{J} \in \mathscr{B}$ such that $\left(x^{(1)}, x^{(2)}, \ldots, x^{(n)}\right)_{\alpha} \subset \tilde{J} \subset \widetilde{D}_{m} \subset \bigcup_{j \in I} \widetilde{D}_{j} \subset T_{F}$. Thus, Definition 3.3(3 $\left.3^{\circ}\right)$ is fulfilled.

Hence, from Definition 3.3, $T_{F}$ is a fuzzy topology for $\mathbb{R}^{n}$ and $\left(\mathbb{R}^{n}, T_{F}\right)$ is a fuzzy topological space, that is, if we set $X=\mathbb{R}^{n}, T=T_{F}$ in Definition 3.3, then the definition holds. Therefore, Definitions 3.5, 3.6 and Property 3.7 can all be applied.

Definition 3.5 (Chang [1, Definition 2.3]). A fuzzy set $\widetilde{U}$ in an FTS $(X, T)$ is a neighborhood of a fuzzy set $\tilde{A}$ if and only if there exists a fuzzy set $\widetilde{O} \in T$ such that $\tilde{A} \subset \tilde{O} \subset \tilde{U}$.

DeFINITION 3.6 (Chang [1, Definition 3]). If a sequence of fuzzy sets $\left\{\tilde{A}_{n}, n=1,2\right.$, $\ldots\}$ is in an FTS $(X, T)$, then this sequence converges to a fuzzy set $\tilde{A}$ if and only if it is eventually contained in each neighborhood of $\widetilde{A}$ (i.e., if $\widetilde{B}$ is any neighborhood of $\widetilde{A}$, there is a positive integer $m$ such that whenever $n \geq m, \widetilde{A}_{n} \subset \widetilde{B}$ ).

Property 3.7. $\left\{\tilde{A}_{n}\right\}$ are increasing fuzzy sets, $\tilde{A}_{1} \subset \tilde{A}_{2} \subset \cdots \subset \tilde{A}$, and

$$
\lim _{n \rightarrow \infty} \mu_{\widetilde{A}_{n}}\left(x^{(1)}, x^{(2)}, \ldots, x^{(n)}\right)=\mu_{\widetilde{A}}\left(x^{(1)}, x^{(2)}, \ldots, x^{(n)}\right)
$$

for all $\left(x^{(1)}, x^{(2)}, \ldots, x^{(n)}\right) \in \mathbb{R}^{n}$. Then the sequence $\left\{\tilde{A}_{n}, n=1,2, \ldots\right\}$ converges to $\tilde{A}$, denoted by $\lim _{n \rightarrow \infty} \tilde{A}_{n}=\tilde{A}$.

Proof. The proof follows from Definition 3.6 easily. 
DEFINITION 3.8. $\bigcup_{\alpha \in[0,1]} O\left(\left(a^{(1,1)}(\alpha), a^{(1,2)}(\alpha)\right), \ldots,\left(a^{(n, 1)}(\alpha), a^{(n, 2)}(\alpha)\right)\right)_{\alpha}\left(\in T_{F}\right)$ is a neighborhood of $\tilde{D}=\bigcup_{\alpha \in[0,1]} D(\alpha)_{\alpha} \in F_{c}$ if and only if for each $\alpha \in[0,1]$, there exists $O\left(\left(a^{(1,1)}(\alpha), a^{(1,2)}(\alpha)\right), \ldots,\left(a^{(n, 1)}(\alpha), a^{(n, 2)}(\alpha)\right)\right)_{\alpha} \in \mathscr{B}$ such that $D(\alpha)_{\alpha} \subset O\left(\left(a^{(1,1)}(\alpha)\right.\right.$, $\left.\left.a^{(1,2)}(\alpha)\right), \ldots,\left(a^{(n, 1)}(\alpha), a^{(n, 2)}(\alpha)\right)\right)_{\alpha}$.

DEFINITION 3.9. In $F_{c}$, the sequence of fuzzy sets $\widetilde{D}_{k}=\bigcup_{\alpha \in[0,1]} D_{k}(\alpha)_{\alpha}, k=1,2, \ldots$, converges to $\tilde{D}=\bigcup_{\alpha \in[0,1]} D(\alpha)_{\alpha}, k=1,2, \ldots\left(\in F_{\alpha}\right)$ if and only if for each neighborhood $\bigcup_{\alpha \in[0,1]} O\left(\left(a^{(1,1)}(\alpha), a^{(1,2)}(\alpha)\right), \ldots,\left(a^{(n, 1)}(\alpha), a^{(n, 2)}(\alpha)\right)\right)_{\alpha}$ of $\tilde{D}$, there exists a natural number $m$ such that whenever $k \geq m, D_{k}(\alpha)_{\alpha} \subset O\left(\left(a^{(1,1)}(\alpha), a^{(1,2)}(\alpha)\right), \ldots,\left(a^{(n, 1)}(\alpha)\right.\right.$, $\left.a^{(n, 2)}(\alpha)\right)_{\alpha}$, denoted by $\lim _{k \rightarrow \infty} \widetilde{D}_{k}=\tilde{D}$.

Since $D \subset \mathbb{R}^{n}$ and $D_{\alpha}\left(\in F D^{*}\right)$ is a one-to-one onto mapping, from Definition 3.9, we can get the following property.

Property 3.10. In $F_{c}$, the sequence of fuzzy sets $\widetilde{D}_{k}=\bigcup_{\alpha \in[0,1]} D_{k}(\alpha)_{\alpha}, k=1,2, \ldots$, converges to $\widetilde{D}=\bigcup_{\alpha \in[0,1]} D(\alpha)_{\alpha}$ if and only if for each $\alpha \in[0,1]$ and every neighborhood $O\left(\left(a^{(1,1)}, a^{(1,2)}\right), \ldots,\left(a^{(n, 1)}, a^{(n, 2)}\right)\right)_{\alpha}$ of $D(\alpha)_{\alpha}$, there exists a natural number $m$ such that whenever $k \geq m, D_{k}(\alpha)_{\alpha} \subset O\left(\left(a^{(1,1)}, a^{(1,2)}\right), \ldots,\left(a^{(n, 1)}, a^{(n, 2)}\right)\right)$ if and only if for each $\alpha \in[0,1]$ and every neighborhood $O\left(\left(a^{(1,1)}, a^{(1,2)}\right), \ldots,\left(a^{(n, 1)}, a^{(n, 2)}\right)\right)_{\alpha}$ of $D(\alpha)$, there exists $m$ such that whenever $k \geq m, D_{k}(\alpha)_{\alpha} \subset O\left(\left(a^{(1,1)}, a^{(1,2)}\right), \ldots,\left(a^{(n, 1)}\right.\right.$, $\left.a^{(n, 2)}\right)$.

The convergency of fuzzy vectors needs the following property.

Property 3.11. For each $\alpha \in[0,1]$, the $\alpha$-cuts $D_{k}(\alpha), E_{k}(\alpha), k=1,2, \ldots, m$, of $\widetilde{D}_{k}$, $\widetilde{E}_{k}$ in $F_{c}$ satisfy the following:

$\left(1^{\circ}\right)\left(D_{k}(\alpha)(+) E_{k}(\alpha)\right)_{\alpha}=D_{k}(\alpha)_{\alpha} \oplus E_{k}(\alpha)_{\alpha}$,

$\left(2^{\circ}\right)\left(D_{k}(\alpha)(-) E_{k}(\alpha)\right)_{\alpha}=D_{k}(\alpha)_{\alpha} \ominus E_{k}(\alpha)_{\alpha}$,

$\left(3^{\circ}\right)$ each $\alpha$-cut of $\bigcup_{k=1}^{m}\left[\widetilde{D}_{k} \oplus \widetilde{E}_{k}\right]$ is $\bigcup_{k=1}^{m}\left[\widetilde{D}_{k}(\alpha)(+) \widetilde{E}_{k}(\alpha)\right]$,

$\left(3^{\circ}-1\right)\left(\bigcup_{k=1}^{m}\left(D_{k}(\alpha)(+) E_{k}(\alpha)\right)\right)_{\alpha}=\bigcup_{k=1}^{m}\left(D_{k}(\alpha)(+) E_{k}(\alpha)\right)_{\alpha}=\bigcup_{k=1}^{m}\left(D_{k}(\alpha)_{\alpha} \oplus E_{k}(\alpha)_{\alpha}\right)=$ $\left(\bigcup_{k=1}^{m} D_{k}(\alpha)_{\alpha}\right) \oplus\left(\bigcup_{k=1}^{m} D_{k}(\alpha)_{\alpha}\right)$,

$\left(3^{\circ}-2\right) \bigcup_{k=1}^{m}\left(\widetilde{D}_{k} \oplus \widetilde{E}_{k}\right)=\left(\bigcup_{k=1}^{m} \widetilde{D}_{k}\right) \oplus\left(\bigcup_{k=1}^{m} \widetilde{E}_{k}\right)$,

$\left(4^{\circ}\right)$ the $\alpha$-cut of $\bigcup_{k=1}^{m}\left(\widetilde{D}_{k} \ominus \widetilde{E}_{k}\right)$ is $\bigcup_{k=1}^{m}\left[D_{k}(\alpha)(-) E_{k}(\alpha)\right]$,

$\left(4^{\circ}-1\right)\left(\bigcup_{k=1}^{m}\left(D_{k}(\alpha)(-) E_{k}(\alpha)\right)\right)_{\alpha}=\bigcup_{k=1}^{m}\left(D_{k}(\alpha)(-) E_{k}(\alpha)\right)_{\alpha}=\bigcup_{k=1}^{m}\left(D_{k}(\alpha)_{\alpha} \ominus E_{k}(\alpha)_{\alpha}\right)=$ $\left(\bigcup_{k=1}^{m} D_{k}(\alpha)_{\alpha}\right) \ominus\left(\bigcup_{k=1}^{m} D_{k}(\alpha)_{\alpha}\right)$,

$\left(4^{\circ}-2\right) \bigcup_{k=1}^{m}\left(\widetilde{D}_{k} \ominus \widetilde{E}_{k}\right)=\left(\bigcup_{k=1}^{m} D_{k}\right) \ominus\left(\bigcup_{k=1}^{m} E_{k}\right)$.

Proof. By extension principle $\left(1^{\circ}\right)$

$$
\begin{aligned}
& \mu_{D_{k}(\alpha)_{\alpha} \oplus E_{k}(\alpha)_{\alpha}}\left(z^{(1)}, z^{(2)}, \ldots, z^{(n)}\right) \\
& =\sup _{\substack{\left.z^{(j)}=x^{(j)}+y^{(j)}\right) \\
j=1,2, \ldots, n}} \mu_{D_{k}(\alpha)_{\alpha}}\left(x^{(1)}, x^{(2)}, \ldots, x^{(n)}\right) \\
& \quad \wedge \mu_{E_{k}(\alpha)_{\alpha}}\left(y^{(1)}, y^{(2)}, \ldots, y^{(n)}\right) \\
& =\sup ^{\left(x^{(1)}, x^{(2)}, \ldots, x^{(n)}\right)} \mu_{D_{k}(\alpha)_{\alpha}}\left(x^{(1)}, x^{(2)}, \ldots, x^{(n)}\right) \\
& \quad \wedge \mu_{E_{k}(\alpha)_{\alpha}}\left(z^{(1)}-x^{(1)}, z^{(2)}-x^{(2)}, \ldots, z^{(n)}-x^{(n)}\right)
\end{aligned}
$$




$$
\begin{aligned}
&=\alpha, \quad \text { if }\left(x^{(1)}, x^{(2)}, \ldots, x^{(n)}\right) \in D_{k}(\alpha), \\
&\left(z^{(1)}-x^{(1)}, z^{(2)}-x^{(2)}, \ldots, z^{(n)}-x^{(n)}\right) \in E_{k}(\alpha), \\
&=\alpha, \quad \text { if }\left(z^{(1)}, z^{(2)}, \ldots, z^{(n)}\right) \in D_{k}(\alpha)(+) E_{k}(\alpha), \\
&=\mu_{\left(D_{k}(\alpha)+E_{k}(\alpha)\right)_{\alpha}}\left(z^{(1)}, z^{(2)}, \ldots, z^{(n)}\right) \quad \forall\left(x^{(1)}, x^{(2)}, \ldots, x^{(n)}\right) \in \mathbb{R}^{n} .
\end{aligned}
$$

$\left(2^{\circ}\right)$ The proof is similar to that of $\left(1^{\circ}\right)$.

$\left(3^{\circ}\right)$ Let $\widetilde{S}_{k}=\widetilde{D}_{k} \oplus \widetilde{E}_{k}$; from $(2.11)$, we have

$$
\bigcup_{k=1}^{m} \tilde{S}_{k}=\bigcup_{k=1}^{m} \bigcup_{\alpha \in[0,1]}\left(D_{k}(\alpha)(+) E_{k}(\alpha)\right)_{\alpha}=\bigcup_{\alpha \in[0,1]} \bigcup_{k=1}^{m}\left(D_{k}(\alpha)(+) E_{k}(\alpha)\right)_{\alpha}
$$

Therefore, the $\alpha$-cut of $\bigcup_{k=1}^{m}\left(\widetilde{D}_{k} \oplus \tilde{E}_{k}\right)=\bigcup_{k=1}^{m} \tilde{S}_{k}$ is $\bigcup_{k=1}^{m} S_{k}(\alpha)=\bigcup_{k=1}^{m}\left(D_{k}(\alpha)(+) E_{k}(\alpha)\right)$. $\left(3^{\circ}-1\right)$ For each $\alpha \in[0,1]$, the subset $\bigcup_{k=1}^{m} S_{k}(\alpha)$ of $\mathbb{R}^{n}$ corresponds to the fuzzy set $\bigcup_{k=1}^{m} S_{k}(\alpha)_{\alpha}=\bigcup_{k=1}^{m}\left(D_{k}(\alpha)(+) E_{k}(\alpha)\right)_{\alpha}$. We first prove

$$
\left(\bigcup_{k=1}^{m} S_{k}(\alpha)\right)_{\alpha}=\bigcup_{k=1}^{m} S_{k}(\alpha)_{\alpha}
$$

We have

$$
\begin{aligned}
\mu_{\cup_{k=1}^{m}}^{m} & S_{k}(\alpha)_{\alpha} \\
\quad & \left(z^{(1)}, z^{(2)}, \ldots, z^{(n)}\right) \\
& \bigvee_{k=1}^{m} \mu_{S_{k}(\alpha)_{\alpha}}\left(z^{(1)}, z^{(2)}, \ldots, z^{(n)}\right) \\
& =\alpha, \quad \text { if }\left(z^{(1)}, z^{(2)}, \ldots, z^{(n)}\right) \in S_{k}(\alpha) \text { for some } k \in\{1,2, \ldots, m\}, \\
& =\alpha, \quad \text { if }\left(z^{(1)}, z^{(2)}, \ldots, z^{(n)}\right) \in \bigcup_{k=1}^{m} S_{k}(\alpha), \\
& =\mu_{\left(\cup_{k=1}^{m} S_{k}(\alpha)\right)_{\alpha}}\left(z^{(1)}, z^{(2)}, \ldots, z^{(n)}\right) \quad \forall\left(z^{(1)}, z^{(2)}, \ldots, z^{(n)}\right) \in \mathbb{R}^{n} .
\end{aligned}
$$

Therefore, $\left(\bigcup_{k=1}^{m} S_{k}(\alpha)\right)_{\alpha}=\bigcup_{k=1}^{m} S_{k}(\alpha)_{\alpha}$. Hence

$$
\left(\bigcup_{k=1}^{m}\left(D_{k}(\alpha)(+) E_{k}(\alpha)\right)\right)_{\alpha}=\bigcup_{k=1}^{m}\left(D_{k}(\alpha)(+) E_{k}(\alpha)\right)_{\alpha} .
$$

For each $\alpha \in[0,1]$ and each $k,\left(1^{\circ}\right)$ holds. Therefore,

$$
\bigcup_{k=1}^{m}\left(D_{k}(\alpha)(+) E_{k}(\alpha)\right)_{\alpha}=\bigcup_{k=1}^{m}\left(D_{k}(\alpha)_{\alpha} \oplus E_{k}(\alpha)_{\alpha}\right)
$$

Finally, we will prove

$$
\bigcup_{k=1}^{m}\left(D_{k}(\alpha)_{\alpha} \oplus E_{k}(\alpha)_{\alpha}\right)=\bigcup_{k=1}^{m}\left(D_{k}(\alpha)_{\alpha}\right) \oplus \bigcup_{k=1}^{m}\left(E_{k}(\alpha)_{\alpha}\right)
$$


We have

$$
\begin{aligned}
\mu_{\cup_{k=1}^{m}}^{m}\left(D_{k}(\alpha)_{\alpha} \oplus E_{k}(\alpha)_{\alpha}\right) & \left(z^{(1)}, z^{(2)}, \ldots, z^{(n)}\right) \\
= & \bigvee_{k=1}^{m} \mu_{D_{k}(\alpha)_{\alpha} \oplus E_{k}(\alpha)_{\alpha}}\left(z^{(1)}, z^{(2)}, \ldots, z^{(n)}\right) \\
= & \bigvee_{k=1}^{m} \sup _{\substack{(j) \\
j=x^{(j)}, 2, \ldots, n}} \mu_{D_{k}(\alpha)_{\alpha}}\left(x^{(1)}, x^{(2)}, \ldots, x^{(n)}\right) \\
& \wedge \mu_{E_{k}(\alpha)_{\alpha}}\left(y^{(1)}, y^{(2)}, \ldots, y^{(n)}\right) \\
= & \bigvee_{k=1}^{m} \bigvee_{\left(y^{(1)}, y^{(2)}, \ldots, y^{(n)}\right)}\left[\mu_{D_{k}(\alpha)_{\alpha}}\left(z^{(1)}-y^{(1)}, z^{(2)}-y^{(2)}, \ldots, z^{(n)}-y^{(n)}\right)\right. \\
= & \left.\wedge \mu_{E_{k}(\alpha)_{\alpha}}\left(y^{(1)}, y^{(2)}, \ldots, y^{(n)}\right)\right] \\
& \bigvee_{\left(y^{(1)}, y^{(2)}, \ldots, y^{(n)}\right)}\left[\mu_{\cup_{k=1}^{m} D_{k}(\alpha)_{\alpha}}\left(z^{(1)}-y^{(1)}, z^{(2)}-y^{(2)}, \ldots, z^{(n)}-y^{(n)}\right)\right. \\
= & \mu_{\left(\cup_{k=1}^{m} D_{k}(\alpha)_{\alpha}\right) \oplus\left(\cup_{k=1}^{m} E_{k}(\alpha)_{\alpha}\right)}\left(z^{(1)}, z^{(2)}, \ldots, z^{(n)}\right) \quad \forall\left(z^{(1)}, z^{(2)}, \ldots, z^{(n)}\right) \in \mathbb{R}^{n} .
\end{aligned}
$$

$\left(3^{\circ}-2\right)$ By decomposition theorem and $\left(3^{\circ}-1\right)$, we have

$$
\begin{aligned}
\bigcup_{k=1}^{m}\left(\widetilde{D}_{k} \oplus \widetilde{E}_{k}\right) & =\bigcup_{k=1}^{m} \bigcup_{\alpha \in[0,1]}\left(D_{k}(\alpha)(+) E_{k}(\alpha)\right)_{\alpha} \\
& =\bigcup_{\alpha \in[0,1]} \bigcup_{k=1}^{m}\left(D_{k}(\alpha)(+) E_{k}(\alpha)\right) \alpha \\
& =\bigcup_{\alpha \in[0,1]}\left[\left(\bigcup_{k=1}^{m} D_{k}(\alpha)_{\alpha}\right) \oplus\left(\bigcup_{k=1}^{m} E_{k}(\alpha)_{\alpha}\right)\right] .
\end{aligned}
$$

Let $\tilde{A}=\bigcup_{k=1}^{m} \widetilde{D}_{k}, \widetilde{B}=\bigcup_{k=1}^{m} \widetilde{E}_{k}$. From (3.9),

$$
\begin{aligned}
A(\alpha)_{\alpha} & =\bigcup_{k=1}^{m} \widetilde{D}_{k}(\alpha)_{\alpha}, \quad B(\alpha)_{\alpha}=\bigcup_{k=1}^{m} \widetilde{E}_{k}(\alpha)_{\alpha}, \quad \forall \alpha \in[0,1], \\
\widetilde{A} \oplus \widetilde{B} & =\bigcup_{\alpha \in[0,1]}[A(\alpha)(+) B(\alpha)]_{\alpha}=\bigcup_{\alpha \in[0,1]}\left[A(\alpha)_{\alpha} \oplus B(\alpha)_{\alpha}\right] \\
& =\bigcup_{\alpha \in[0,1]}\left[\left(\bigcup_{k=1}^{m} D_{k}(\alpha)_{\alpha}\right) \oplus\left(\bigcup_{k=1}^{m} E_{k}(\alpha)_{\alpha}\right)\right] .
\end{aligned}
$$


From (3.15), (3.17), we have

$$
\begin{aligned}
\bigcup_{k=1}^{m}\left(\widetilde{D}_{k} \oplus \widetilde{E}_{k}\right) & =\bigcup_{\alpha \in[0,1]}\left[\left(\bigcup_{k=1}^{m} D_{k}(\alpha)_{\alpha}\right) \oplus\left(\bigcup_{k=1}^{m} E_{k}(\alpha)_{\alpha}\right)\right] \\
& =\left(\bigcup_{k=1}^{m} \widetilde{D}_{k}\right) \oplus\left(\bigcup_{k=1}^{m} \widetilde{E}_{k}\right) .
\end{aligned}
$$

Properties $\left(4^{\circ}\right),\left(4^{\circ}-1\right)$, and $\left(4^{\circ}-2\right)$ can be proved similarly as $\left(3^{\circ}\right),\left(3^{\circ}-1\right)$, and $\left(3^{\circ}-2\right)$.

Property 3.12. $\tilde{D}_{k} \in F_{c}, k=1,2, \ldots, m$, and $q \neq 0$; then

$\left(1^{\circ}\right)$ the $\alpha$-cut of $\bigcup_{k=1}^{m}\left(q_{1} \odot \tilde{D}_{k}\right)$ is $\bigcup_{k=1}^{m}\left(q(\cdot) D_{k}(\alpha)\right)$,

$\left(2^{\circ}\right) \bigcup_{k=1}^{m}\left(q(\odot) D_{k}(\alpha)\right)_{\alpha}=q_{1} \odot\left(\bigcup_{k=1}^{m} D_{k}(\alpha)_{\alpha}\right)$,

$\left(3^{\circ}\right) \bigcup_{k=1}^{m}\left(q_{1} \odot \widetilde{D}_{k}\right)=q_{1} \odot\left(\bigcup_{k=1}^{m} \widetilde{D}_{k}\right)$.

Proof. The proof goes on the lines of the proof of Property 3.11.

Property 3.13. $\widetilde{D}_{m}, \widetilde{E}_{m}, \widetilde{D}, \widetilde{E} \in F_{c}, m=1,2, \ldots$, and $\lim _{m \rightarrow \infty} \widetilde{D}_{m}=\widetilde{D}, \lim _{m \rightarrow \infty} \widetilde{E}_{m}=\widetilde{E}$, then

$\left(1^{\circ}\right) \lim _{m \rightarrow \infty}\left(\widetilde{D}_{m} \oplus \widetilde{E}_{m}\right)=\widetilde{D} \oplus \widetilde{E}=\lim _{m \rightarrow \infty}\left(\widetilde{D}_{m}\right) \oplus \lim _{m \rightarrow \infty}\left(\widetilde{E}_{m}\right)$,

$\left(2^{\circ}\right) \lim _{m \rightarrow \infty}\left(\widetilde{D}_{m} \ominus \widetilde{E}_{m}\right)=\widetilde{D} \ominus \widetilde{E}=\lim _{m \rightarrow \infty}\left(\widetilde{D}_{m}\right) \ominus \lim _{m \rightarrow \infty}\left(\widetilde{E}_{m}\right)$,

$\left(3^{\circ}\right) \lim _{m \rightarrow \infty}\left(k_{1} \odot \widetilde{D}_{m}\right)=k_{1} \odot \widetilde{D}=k_{1} \odot\left(\lim _{m \rightarrow \infty}\left(\widetilde{D}_{m}\right)\right), k \neq 0$.

Proof. $\left(1^{\circ}\right)$ Since $\lim _{m \rightarrow \infty} \widetilde{D}_{m}=\widetilde{D}, \lim _{m \rightarrow \infty} \widetilde{E}_{m}=\widetilde{E}$, by Property 3.10, for each $\alpha \in$ $[0,1]$ and every neighborhood $O\left(\left(a^{(1,1)}, a^{(1,2)}\right), \ldots,\left(a^{(n, 1)}, a^{(n, 2)}\right)\right)$ of $D(\alpha)$, there exists a natural number $m^{(1)}$ such that when $k \geq m^{(1)}, D_{k}(\alpha) \subset O\left(\left(a^{(1,1)}, a^{(1,2)}\right), \ldots,\left(a^{(n, 1)}\right.\right.$, $\left.\left.a^{(n, 2)}\right)\right)$. Also, for every neighborhood $O\left(\left(b^{(1,1)}, b^{(1,2)}\right), \ldots,\left(b^{(n, 1)}, b^{(n, 2)}\right)\right)$ of $E(\alpha)$, there exists a natural number $m^{(2)}$ such that when $k \geq m^{(2)}, E_{k}(\alpha) \subset O\left(\left(b^{(1,1)}, b^{(1,2)}\right), \ldots\right.$, $\left.\left(\boldsymbol{b}^{(n, 1)}, \boldsymbol{b}^{(n, 2)}\right)\right)$.

Let $m=\max \left(m^{(1)}, m^{(2)}\right)$. Then, for each $\alpha \in[0,1]$, when $k \geq m$, by (3.2), we have $D_{k}(\alpha)(+) E_{k}(\alpha) \subset O\left(\left(a^{(1,1)}+b^{(1,1)}, a^{(1,2)}+b^{(1,2)}\right), \ldots,\left(a^{(n, 1)}+b^{(n, 1)}, a^{(n, 2)}+b^{(n, 2)}\right)\right)(\in$ $\left.T_{F}\right)$, and $O\left(\left(a^{(1,1)}+b^{(1,1)}, a^{(1,2)}+b^{(1,2)}\right), \ldots,\left(a^{(n, 1)}+b^{(n, 1)}, a^{(n, 2)}+b^{(n, 2)}\right)\right)$ is the neighborhood of $D(\alpha)(+) E(\alpha)$. By decomposition theorem,

$$
\begin{gathered}
\widetilde{D}_{k} \oplus \widetilde{E}_{k}=\bigcup_{\alpha \in[0,1]}\left[D_{k}(\alpha)+E_{k}(\alpha)\right]_{\alpha}, \\
\tilde{D} \oplus \widetilde{E}=\bigcup_{\alpha \in[0,1]}[D(\alpha)+E(\alpha)]_{\alpha} .
\end{gathered}
$$

Hence, by Property 3.10, we have $\lim _{m \rightarrow \infty} \widetilde{D}_{m} \oplus \widetilde{E}_{m}=\widetilde{D} \oplus \widetilde{E}$.

Properties $\left(2^{\circ}\right)$ and $\left(3^{\circ}\right)$ can be proved the same way as $\left(1^{\circ}\right)$.

Property 3.14. $\widetilde{D}_{k}, \widetilde{E}_{k}, \widetilde{D}, \widetilde{E} \in F_{c}, k=1,2, \ldots$, and

$$
\begin{aligned}
& \lim _{m \rightarrow \infty} \mu_{\cup_{k=1}^{m} \widetilde{D}_{k}}\left(x^{(1)}, x^{(2)}, \ldots, x^{(n)}\right)=\mu_{\widetilde{D}}\left(x^{(1)}, x^{(2)}, \ldots, x^{(n)}\right), \\
& \lim _{m \rightarrow \infty} \mu_{\cup_{k=1}^{m} \widetilde{E}_{k}}\left(x^{(1)}, x^{(2)}, \ldots, x^{(n)}\right) \\
& =\mu_{\widetilde{E}}\left(x^{(1)}, x^{(2)}, \ldots, x^{(n)}\right) \quad \forall\left(x^{(1)}, x^{(2)}, \ldots, x^{(n)}\right) \in \mathbb{R}^{n}, \\
& \mu_{\cup_{k=1}^{m} \widetilde{D}_{k}} \subset \widetilde{D}, \quad \mu_{\cup_{k=1}^{m} \widetilde{E}_{k}} \subset \widetilde{E}, \quad \forall m=1,2, \ldots,
\end{aligned}
$$


then

$\left(1^{\circ}\right) \lim _{m \rightarrow \infty} \bigcup_{k=1}^{m}\left(\widetilde{D}_{k} \oplus \tilde{E}_{k}\right)=\tilde{D} \oplus \widetilde{E}=\left(\lim _{m \rightarrow \infty} \bigcup_{k=1}^{m} \widetilde{D}_{k}\right) \oplus\left(\lim _{m \rightarrow \infty} \bigcup_{k=1}^{m} \widetilde{E}_{k}\right)$,

$\left(2^{\circ}\right) \lim _{m \rightarrow \infty} \bigcup_{k=1}^{m}\left(\widetilde{D}_{k} \ominus \widetilde{E}_{k}\right)=\tilde{D} \ominus \widetilde{E}=\left(\lim _{m \rightarrow \infty} \bigcup_{k=1}^{m} \widetilde{D}_{k}\right) \ominus\left(\lim _{m \rightarrow \infty} \bigcup_{k=1}^{m} \widetilde{E}_{k}\right)$,

$\left(3^{\circ}\right)$ when $q \neq 0, \lim _{m \rightarrow \infty} \bigcup_{k=1}^{m}\left(q_{1} \odot \widetilde{D}_{k}\right)=q_{1} \odot \widetilde{D}$.

Proof. $\left(1^{\circ}\right)$ Since $\widetilde{D}_{1} \subset \widetilde{D}_{1} \cup \widetilde{D}_{2} \subset \cdots \subset \bigcup_{k=1}^{m} \widetilde{D}_{k} \subset \cdots \subseteq \widetilde{D}$ and

$$
\lim _{m \rightarrow \infty} \mu_{\cup_{k=1}^{m} \widetilde{D}_{k}}\left(x^{(1)}, x^{(2)}, \ldots x^{(n)}\right)=\mu_{\widetilde{D}}\left(x^{(1)}, x^{(2)}, \ldots, x^{(n)}\right)
$$

for all $\left(x^{(1)}, x^{(2)}, \ldots, x^{(n)}\right) \in \mathbb{R}^{n}$, hence, by Property 3.7, we have $\lim _{m \rightarrow \infty} \bigcup_{k=1}^{m} \widetilde{D}_{k}=\tilde{D}$. Similarly, $\lim _{m \rightarrow \infty} \bigcup_{k=1}^{m} \widetilde{E}_{k}=\widetilde{E}$. By Property $3.11\left(3^{\circ}-2\right)$,

$$
\bigcup_{k=1}^{m}\left(\widetilde{D}_{k} \oplus \widetilde{E}_{k}\right)=\left(\bigcup_{k=1}^{m} \widetilde{D}_{k}\right) \oplus\left(\bigcup_{k=1}^{m} \widetilde{E}_{k}\right)
$$

From Property 3.13(1 ${ }^{\circ}$,

$$
\lim _{m \rightarrow \infty} \bigcup_{k=1}^{m}\left(\widetilde{D}_{k} \oplus \widetilde{E}_{k}\right)=\left(\lim _{m \rightarrow \infty} \bigcup_{k=1}^{m}\left(\widetilde{D}_{k}\right)\right) \oplus\left(\lim _{m \rightarrow \infty} \bigcup_{k=1}^{m}\left(\widetilde{E}_{k}\right)\right)=\widetilde{D} \oplus \widetilde{E},
$$

and $\left(2^{\circ}\right),\left(3^{\circ}\right)$ can be proved as $\left(1^{\circ}\right)$.

Next, we will discuss the convergency of the fuzzy vectors in SFR.

Property 3.15. For ${\widetilde{\widetilde{D}_{m}}}_{\tilde{E}_{m}}, \widetilde{D}, \widetilde{E} \in F_{c}, m=1,2, \ldots, \lim _{m \rightarrow \infty} \widetilde{D}_{m}=\widetilde{D}, \lim _{m \rightarrow \infty} \widetilde{E}_{m}=\widetilde{E}$, then the fuzzy vectors $\overrightarrow{\widetilde{E}_{m} \widetilde{D}_{m}}, m=1,2, \ldots$, converge to the fuzzy vectors $\overrightarrow{\widetilde{E} \widetilde{D}}$.

Proof. Since $\overrightarrow{\widetilde{E}_{m} \widetilde{D}_{m}}=\widetilde{D}_{m} \ominus \widetilde{E}_{m}, \overrightarrow{\widetilde{E} \widetilde{D}}=\widetilde{D} \ominus \widetilde{E}$, then, by Property $3.13\left(2^{\circ}\right)$,

$$
\lim _{m \rightarrow \infty} \overrightarrow{\widetilde{E}_{m} \widetilde{D}_{m}}=\widetilde{D} \ominus \widetilde{E}=\overrightarrow{\widetilde{E} \widetilde{D}}
$$

ProperTy 3.16. $\widetilde{D}_{k}, \widetilde{E}_{k}, \widetilde{D}, \widetilde{E} \in F_{c}, k=1,2 \ldots$; let $\widetilde{Q}_{m}=\bigcup_{k=1}^{m} \widetilde{D}_{k}, \widetilde{S}_{m}=\bigcup_{k=1}^{m} \widetilde{E}_{k}$, and let $\lim _{m \rightarrow \infty} \mu_{\widetilde{Q}_{m}}\left(x^{(1)}, x^{(2)}, \ldots, x^{(n)}\right)=\mu_{\widetilde{D}}\left(x^{(1)}, x^{(2)}, \ldots, x^{(n)}\right)$ and $\lim _{m \rightarrow \infty} \mu_{\widetilde{S}_{m}}\left(x^{(1)}, x^{(2)}, \ldots\right.$, $\left.x^{(n)}\right)=\mu_{\widetilde{E}}\left(x^{(1)}, x^{(2)}, \ldots, x^{(n)}\right)$ for all $\left(x^{(1)}, x^{(2)}, \ldots, x^{(n)}\right) \in \mathbb{R}^{n}$, and $\widetilde{Q}_{m} \subset \widetilde{D}, \widetilde{S}_{m} \subset \widetilde{E}$. Then the sequence of fuzzy vectors $\overrightarrow{\widetilde{S}_{m} \widetilde{Q}_{m}}, m=1,2, \ldots$, converges to the fuzzy vector $\overrightarrow{\widetilde{E} \tilde{D}}$.

Proof. Similar to Property 3.14, $\lim _{m \rightarrow \infty} \bigcup_{k=1}^{m} \widetilde{D}_{k}=\widetilde{D}$ and $\lim _{m \rightarrow \infty} \bigcup_{k=1}^{m} \widetilde{E}_{k}=\widetilde{E}$. By Property 3.13(2 $), \lim _{m \rightarrow \infty} \overrightarrow{\widetilde{S}_{m} \widetilde{Q}_{m}}=\left(\lim _{m \rightarrow \infty} \bigcup_{k=1}^{m} \widetilde{D}_{k}\right) \ominus\left(\lim _{m \rightarrow \infty} \bigcup_{k=1}^{m} \widetilde{E}_{k}\right)=\widetilde{D} \ominus \widetilde{E}=\overrightarrow{\widetilde{E} \widetilde{D}}$. For convenience, we denote $\left(q_{1}^{(1)} \odot \overrightarrow{\widetilde{E}_{1} \widetilde{D}_{1}}\right) \oplus\left(q_{1}^{(2)} \odot \overrightarrow{\widetilde{E}_{2} \widetilde{D}_{2}}\right) \oplus \cdots \oplus\left(q_{1}^{(r)} \odot \overrightarrow{\widetilde{E}_{r} \widetilde{D}_{r}}\right)$ by $\sum_{k=1}^{r} \oplus$ $\left(q_{1}^{(k)} \odot \overrightarrow{\widetilde{E}_{k} \widetilde{D}_{k}}\right)$.

Property 3.17. $\widetilde{D}_{m, k}, \widetilde{E}_{m, k}, \widetilde{D}_{k}, \widetilde{E}_{k} \in F_{c}, m=1,2, \ldots, k=1,2, \ldots, r$, and for each $k \in$ $\{1,2, \ldots, r\}, \lim _{m \rightarrow \infty} \widetilde{D}_{k, m}=\widetilde{D}_{k}, \lim _{m \rightarrow \infty} \widetilde{D}_{k, m}=\widetilde{D}_{k}, q^{k} \neq 0$. The sequence of the fuzzy vectors $\sum_{k=1}^{r} \oplus\left(q_{1}^{(k)} \odot \overrightarrow{\widetilde{E}_{m, k} \widetilde{D}_{m, k}}\right), m=1,2, \ldots$, converges to the fuzzy vector $\sum_{k=1}^{r} \oplus\left(q_{1}^{(k)} \odot\right.$ $\overrightarrow{\widetilde{E}_{k} \widetilde{D}_{k}}$. 
Proof. Since $\sum_{k=1}^{r} \oplus\left(q_{1}^{(k)} \odot \overrightarrow{\widetilde{E}_{m, k} \widetilde{D}_{m, k}}\right)=\sum_{k=1}^{r} \oplus\left(q_{1}^{(k)} \odot\left(\widetilde{D}_{m, k} \ominus \widetilde{E}_{m, k}\right)\right), m=1,2, \ldots$, for each $k$, by Property 3.13(2॰), $\lim _{m \rightarrow \infty} \widetilde{D}_{m, k} \ominus \widetilde{E}_{m, k}=\widetilde{D}_{k} \ominus \widetilde{E}_{k}$. By Property $3.13\left(1^{\circ}\right)$, $\left(3^{\circ}\right)$, we have

$$
\begin{aligned}
\lim _{m \rightarrow \infty} & \sum_{k=1}^{r} \oplus\left(q_{1}^{(k)} \odot\left(\widetilde{D}_{m, k} \ominus \widetilde{E}_{m, k}\right)\right) \\
& =\sum_{k=1}^{r} \oplus\left(q_{1}^{(k)} \odot\left(\widetilde{D}_{k} \ominus \widetilde{E}_{k}\right)\right)=\sum_{k=1}^{r} \oplus\left(q_{1}^{(k)} \odot \overrightarrow{\widetilde{E}_{k} \widetilde{D}_{k}}\right) .
\end{aligned}
$$

EXAMPLE 3.18. Consider the fuzzy vectors $\lim _{m \rightarrow \infty} \overrightarrow{\widetilde{Q}_{m}}$ in Example 2.11. Let

$$
\mu_{\tilde{Z}}(x, y)= \begin{cases}1-(x-10)^{2}-(y-30)^{2}, & \text { if }(x-10)^{2}+(y-30)^{2} \leq 1 \\ 0, & \text { elsewhere }\end{cases}
$$

We will prove $\lim _{m \rightarrow \infty} \tilde{Z}_{m}=\tilde{Z}$. Since $C((10,30), 1+1 / m) \subset C((10,30), 1+1 /(m-1))$ and for any $(x, y) \in \mathbb{R}^{2}$, the following holds:

$$
\begin{aligned}
& \frac{1}{(1+1 / m)^{2}}\left[\left(1+\frac{1}{m}\right)^{2}-(x-10)^{2}-(y-30)^{2}\right] \\
& \quad \leq \frac{1}{(1+1 /(m-1))^{2}}\left[\left(1+\frac{1}{m-1}\right)^{2}-(x-10)^{2}-(y-30)^{2}\right],
\end{aligned}
$$

therefore, $\mu_{\tilde{Z}_{m}}(x, y) \leq \mu_{\tilde{Z}_{m-1}}(x, y)$ for all $(x, y) \in \mathbb{R}^{2}$, and hence $\tilde{Z}_{1} \supset \tilde{Z}_{2} \supset \cdots \supset \tilde{Z}_{m} \supset$ $\cdots \supset \tilde{Z}$, and obviously, $\lim _{m \rightarrow \infty} \mu_{\tilde{Z}_{m}}(x, y)=\mu_{\tilde{Z}}(x, y)$ for all $(x, y) \in \mathbb{R}^{2}$. Let $\tilde{Z}_{m}^{\prime}, \tilde{Z}^{\prime}$ be the complement fuzzy sets of $\widetilde{Z}_{m}, \tilde{Z}$, respectively. We have $\lim _{m \rightarrow \infty} \mu_{\tilde{Z}_{m}^{\prime}}(x, y)=$ $\mu_{\tilde{Z}^{\prime}}(x, y)$ for all $(x, y) \in \mathbb{R}^{2}$ and $\tilde{Z}_{1}^{\prime} \subset \tilde{Z}_{2}^{\prime} \subset \cdots \subset \tilde{Z}_{m}^{\prime} \subset \cdots \subset \tilde{Z}^{\prime}$. By Property 3.7 , $\lim _{m \rightarrow \infty} \tilde{Z}+m^{\prime}=\tilde{Z}^{\prime}$. Thus, $\lim _{m \rightarrow \infty} \tilde{Z}_{m}=\tilde{Z}$. Therefore, from Property $3.15, \lim _{m \rightarrow \infty} \overrightarrow{\widetilde{Q} \tilde{Z}_{m}}$ $=\overrightarrow{\widetilde{Q}}$. Thus, the membership function of $\overrightarrow{\widetilde{Q} \widetilde{Z}}$ is

$$
\begin{aligned}
\mu \underset{\widetilde{Q} \widetilde{Z}}{ }(x, y)= & \mu_{\tilde{Z} \ominus \widetilde{Q}}(x, y) \\
= & \sup _{\substack{x=x^{(1)}-y^{(1)} \\
y=x^{(2)}-y^{(2)}}} \mu_{\widetilde{Z}}\left(x^{(1)}, x^{(2)}\right) \wedge \mu_{\widetilde{Q}}\left(y^{(1)}, y^{(2)}\right) \\
= & \mu_{\widetilde{Z}}(x+1, y+2) \\
= & \begin{cases}1-(x-9)^{2}-(y-28)^{2}, & \text { if }(x-9)^{2}-(y-28)^{2} \leq 1, \\
0, & \text { elsewhere. }\end{cases}
\end{aligned}
$$

In the crisp case, starting from $Q=(1,2)$, aiming at $Z=(10,30)$, we could have the vector $\overrightarrow{Q Z}=(9,28)$. The grade of membership of $\overrightarrow{Q Z}$ which belongs to the fuzzy vector $\overrightarrow{\widetilde{Q} \widetilde{Z}}$ is $\mu_{\widetilde{Q} \widetilde{Z}}(9,28)=1$, that is, the grade of membership function of the fuzzy vector $\overrightarrow{\widetilde{P} \widetilde{Z}}$ for the crisp vector $\overrightarrow{P S}$ is 1 , and the point $R=(9.5,29.5)$ is in the circle of center $(9,28)$ and radius 1 . The crisp vector of $Q$ to $\mathbb{R}$ is $\overrightarrow{Q R}=(8.5,27.5)$. The grade of membership 
function of $\overrightarrow{\widetilde{Q} \widetilde{Z}}$ is $\mu_{\overrightarrow{\widetilde{Q}} \tilde{Z}}(8.5,27.5)=0.5$, that is, the grade of membership function of the fuzzy vector $\overrightarrow{\widetilde{P}} \widetilde{Z}$ for the crisp vector $\overrightarrow{Q R}$ is 0.5 .

\section{REFERENCES}

[1] C. L. Chang, Fuzzy topological spaces, J. Math. Anal. Appl. 24 (1968), 182-190.

[2] A. Kaufmann and M. M. Gupta, Introduction to Fuzzy Arithmetic. Theory and Applications, Van Nostrand Reinhold, New York, 1991.

[3] P. M. Pu and Y. M. Liu, Fuzzy topology. I. Neighborhood structure of a fuzzy point and MooreSmith convergence, J. Math. Anal. Appl. 76 (1980), no. 2, 571-599.

[4] K. Wu, Extension of $n$-dimensional Euclidean vector space $E^{n}$ over $\mathbb{R}$ to pseudo-fuzzy vector space over $F_{p}^{1}(1)$, Int. J. Math. Math. Sci. 2003 (2003), no. 37, 2349-2373.

Kweimei Wu: Department of Mathematics, National Taiwan University, Taipei, Taiwan 


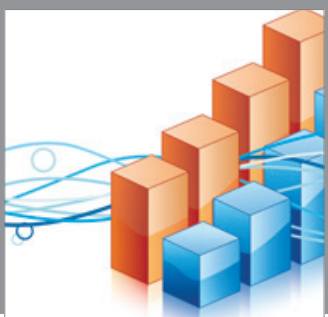

Advances in

Operations Research

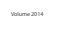

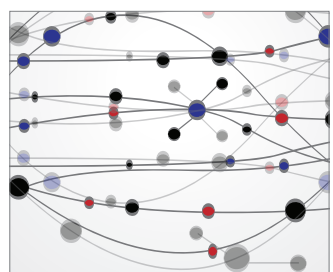

\section{The Scientific} World Journal
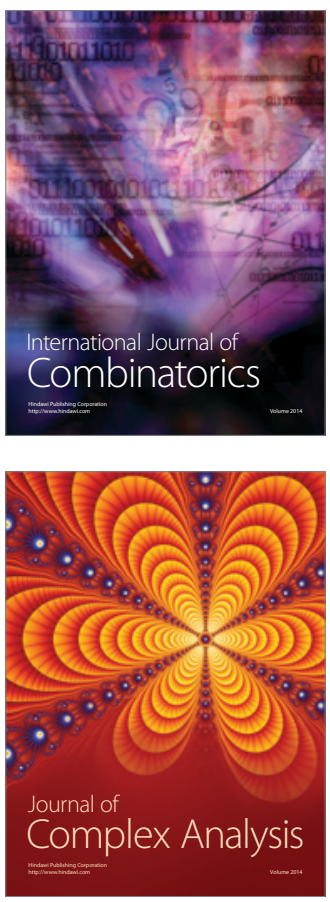

International Journal of

Mathematics and

Mathematical

Sciences
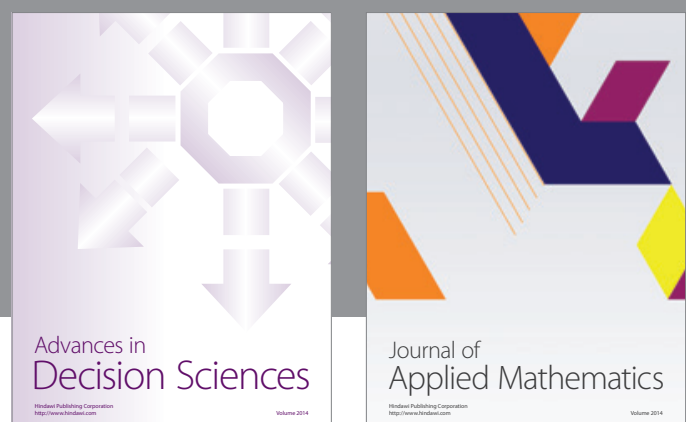

Journal of

Applied Mathematics
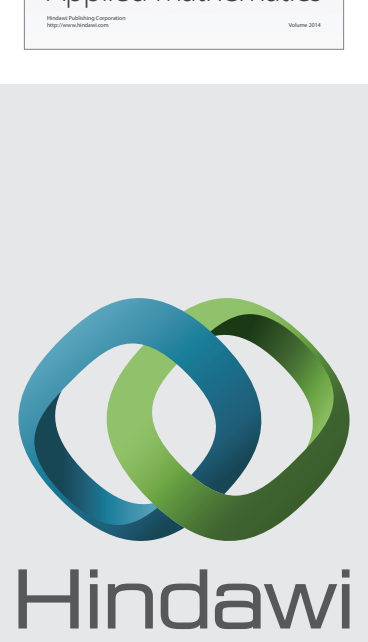

Submit your manuscripts at http://www.hindawi.com
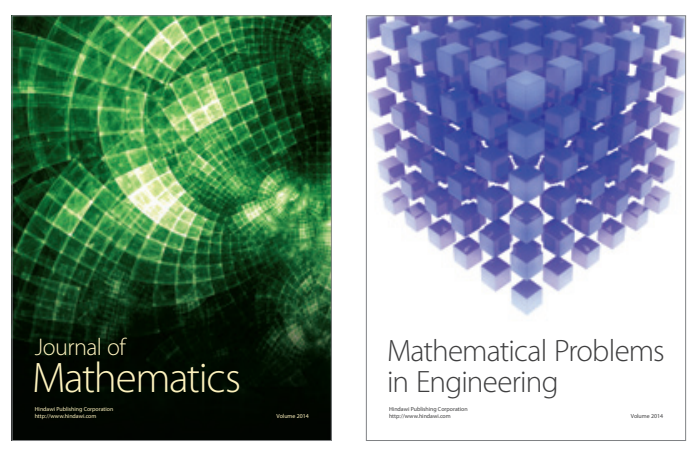

Mathematical Problems in Engineering
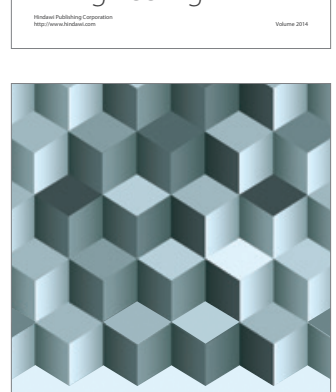

Journal of

Function Spaces
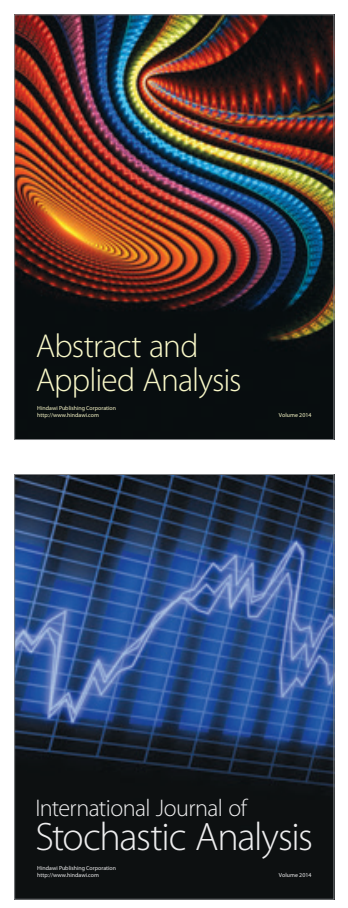

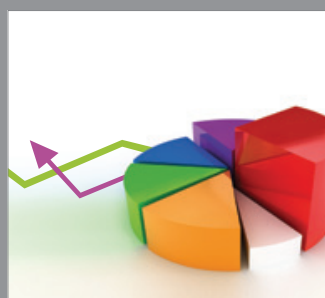

ournal of

Probability and Statistics

Promensencen
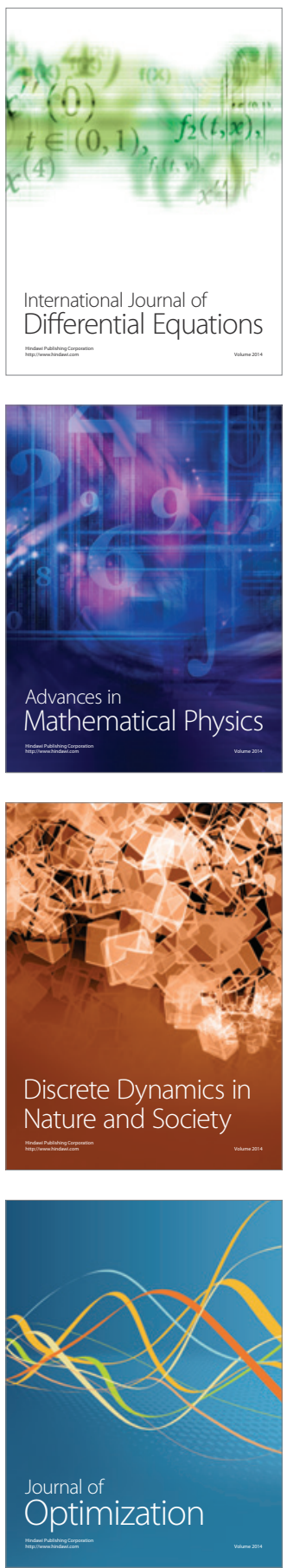